\title{
A Multi-agent System for Cross-Organizational Workflows Management Based on Process-View*
}

\author{
Ping Jiang, Xinyu Shao, Liang Gao, and Zhenfeng Yang \\ Department of Industrial \& Manufacturing System Engineering, \\ Huazhong University of Science \& Technology, 430074, Wuhan, China \\ gaoliang@mail.hust.edu.cn
}

\begin{abstract}
Workflow interoperability across enterprises boundaries presents a significant challenge recently. Process-view provides a promising way to crossorganizational workflows design. A multi-agent system combined with Petri net based process-view approach is proposed to facilitate cross-organizational workflows management. Within this multi-agent system, a Petri net algebra model and a character string mapping algorithm are proposed to automate the mapping from a Petri net workflow model to a process-view workflow model; the synchronization points are added to integrated process-view workflow models to coordinate the execution of cross-organizational workflow instances.
\end{abstract}

Keywords: Cross-organizational workflows management; Process-view; Petri net; Multi-agent system; Mapping.

\section{Introduction}

The existing Workflow Management Systems (WMS) are commonly developed for stable and long-running business processes in individual enterprises, which are always rigid and centralized. But in cross-organizational business collaboration, cooperative processes are highly dynamic and the execution of collaborative activities are distributed, the required WMS should support dynamic and flexible business collaboration across enterprises boundaries while maintaining privacy and autonomy of participant enterprises. Facilitating interoperability among existing WMS provides a promising way for realizing efficient cross-organizational workflows collaboration, which avoids spending time and cost on developing new WMS.

A process-view is an abstract process derived from an implemented base process [1]. In the process-view approach, an enterprise can realize business processes integration with various partner enterprises by reusing its internal processes, which saves much time to remodeling. Our previous work [2] proposed a Petri net based process-view approach for workflow modeling in virtual enterprises. This paper proposes a Multi-Agent System (MAS) combined with the previous approach to facilitate interoperability among existing WMS.

* Supported by the National Basic Research Program 973 of China (No. 2004CB719405). 


\section{Background and Related Work}

The Workflow Management Coalition (WfMC) has developed specifications to enable interoperability between heterogeneous workflow systems [3]. W.M.P. van der Aalst [4] adopted Petri net to model interorganizational workflow. Liu and Shen [1] proposed an order-preserving process-view approach to business-to-business (B2B) workflow interoperability. Martin A. et al [5] utilized Petri net refinement paradigm to simplify the handling of complex and large-scale workflows. The MAS offers a distributed and open platform architecture for dynamically changing systems. In this paper, we propose a MAS combined with the Petri net based process-view approach to manage cross-organizational workflows, which can automate the mapping from a Petri net workflow model to a process-view workflow model and coordinate the execution of cross-organizational workflow instances.

\section{A MAS for Cross-Organizational Workflows Management}

The MAS combined with Petri net based process-view approach (PVMAS) is described in Fig. 1. It provides a mediator architecture to facilitate distributed WMS interoperability.

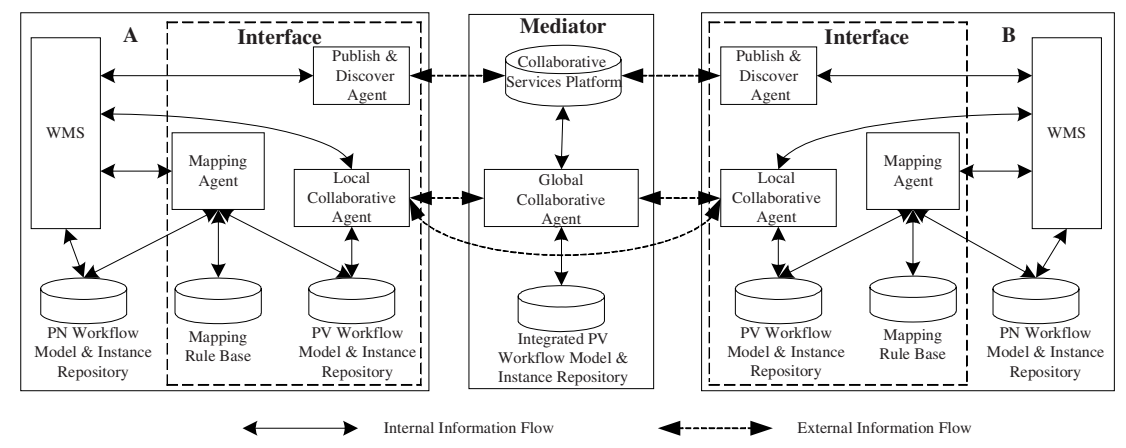

Fig. 1. The PVMAS for cross-organizational workflows management

\subsection{Mapping Agent}

The Mapping Agent automates the mapping from a Petri Net (PN) workflow model to a Process-View (PV) workflow model. A PN algebra model is proposed to automate the mapping.

Definition 1. A PN algebra model is a character string $\mathrm{L}$ consists of $\mathrm{P} i, \mathrm{~A} j, \mathrm{~T} k, \mathrm{Lm},+$, ,- *, /, [ ], ( ) and F, Where:

(1) $\mathrm{P} i, \mathrm{Aj}, \mathrm{T} k$ and $\mathrm{Lm}$ are the starting and ending characters representing base place, abstract place, base transition and logic transition respectively, where $i, j, k$ and $m$ are the sequence number;

(2) $+,-,, *, /$ represent the order structures of Or-Join, Or-Split, And-Join and AndSplit respectively; 
(3) [ ] represents the Loop structure, the places and transitions included in it are executed repeatedly;

(4) ( ) represents the parallel branch;

(5) F represents nothing.

The Mapping Agent can realize the automation of mapping by intelligently processing character strings. We use " \{\} " to denote the sub-models must be hidden. Fig. 2 describes the mapping, and the mapping steps are as follows:

(1) Build the PN workflow model described in Fig. 2.(a);

(2) Get the corresponding PN algebra model L described in Fig. 2.(b);

(3) Select the sub-models needed to be hidden in the PN workflow model. In Fig. 2.(c), the sub-models $\{\mathrm{P} 02, \mathrm{~T} 02, \mathrm{P} 03\}$ and $\{\mathrm{T} 05, \mathrm{P} 06, \mathrm{~T} 06, \mathrm{P} 07\}$ surrounded by curving dashed line are the selected ones;

(4) Add " \{\} " to the PN algebra model to denote the selected sub-models, and get the middle character string L' described in Fig. 2.(d);

(5) Process L' by character string mapping algorithm, the PN algebra model L' of the PV workflow model can be obtained described in Fig. 2.(e), in which submodels $\{\mathrm{P} 02-(\mathrm{T} 02 \mathrm{P} 03)\}$ and $\{[\mathrm{T} 05 \mathrm{P} 06 \mathrm{~T} 06 \mathrm{P} 07]\}$ in $\mathrm{L}^{\prime}$ are replaced by abstract places $\mathrm{A} 01$ and $\mathrm{A} 02$ respectively;

(6) Get the PV workflow model from the character string L“ described in Fig. 2.(f).

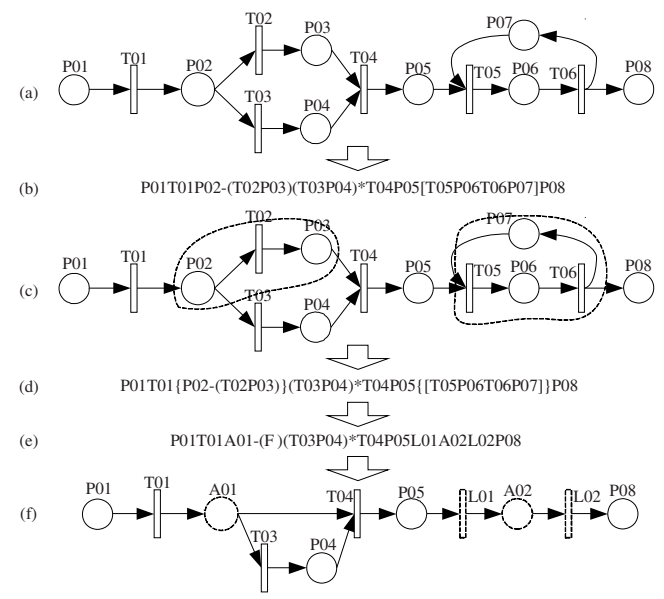

Fig. 2. The mapping from a PN workflow model to a PV workflow model.(a) The PN workflow model; (b) the PN algebra model L; (c) the PN workflow model with sub-models needed to be hidden; (d) the PN algebra model L'; (e) the PN algebra model L" "; (f) the PV workflow model.

The core of the character string mapping algorithm is graph reducing theory. The main procedures of the character string mapping algorithm are as follows:

input $>$ PN1; $\quad$ // Input initial character string

PN1 = find_nodes(PN1); // Find out all nodes

out_in_way(PN2); // Search the split and join nodes 
creat_graph(PN2); // Transform the character string to a matrix

combine_graph(PN1); // Replace the nodes needed to be hidden with necessary abstract place nodes

insert_L( ); // Add some necessary logic transition nodes

ans = output_graph( ); // Transform the matrix into a character string

output(ans). $\quad$ // Output the last character string

\subsection{Local and Global Collaborative Agent}

In order to coordinate the execution of cross-organizational workflow instances, we designate the synchronization points (SP) in the integrated PV workflow models.

Definition 2. SP are some base transitions in integrated PV workflow models, which can facilitate the exchange of workflow status information and the coordination of the workflow progress among the corresponding workflow instances.

When the integrated PV workflow instance execute to SP, the SP will trigger the Global Collaborative Agent to inquire the status information of corresponding PV workflow instances from Local Collaborative Agents of participant enterprises. If there exist some delayed or abnormal information, the responsible Local Collaborative Agents will be triggered to synchronize the execution of corresponding PN workflow instances of participant enterprises.

\section{Conclusion}

The PVMAS for cross-organizational workflows management has the following advantages: Different WMS can finish a common business collaboratively across enterprises boundaries and keep autonomy; enterprises can adopt different PV workflow models mapped from the same PN workflow model to quickly construct cross-organizational workflows.

Our future work will mainly address two themes. First, extending PN to Timed Colored Petri Net (TCPN) to enhance the modeling ability of our system. Second, developing the PVMAS and validating the availability of the system.

\section{References}

1. Duen-Ren L., Minxin S.: Workflow modeling for virtual processes: an order-preserving process-view approach. Information Systems 28 (2003) 505-532

2. Xinyu S., Ping J., Haobo Q., Liang G.: Workflow Modeling for Virtual Enterprise: a Petri Net Based Process-View Approach. Proceedings of the 10th International Conference on Computer Supported Cooperative Work in Design 2(2006) 1213-1218

3. Workflow Management Coalition.: Interface 1: Process definition interchange process model. Technical report WfMC TC-1016-P (1998)

4. Van der Aalst. W.M.P.: Loosely coupled interorganizational workflows: modeling and analyzing crossing organizational boundaries. Information \& Management 37 (2000) 67-75

5. Martin A., Sergei G., Andreas, H., Hans-Werner, P.: Using High-Level Petri Nets for Hierarchical Grid Workflows. Proceedings of the Second IEEE International Conference on e-Science and Grid Computing (2006) 